\title{
Intraoperative severe suprachoroidal air as a complication of 23-gauge vitrectomy combined with air-fluid exchange
}

This article was published in the following Dove Press journal: International Medical Case Reports Journal

\author{
Chien-Jen Lin' \\ Kai-Ling Peng ${ }^{2}$ \\ 'Department of Radiology, Chi Mei \\ Medical Center, Tainan City, Taiwan; \\ ${ }^{2}$ Department of Ophthalmology, \\ Chi Mei Medical Center, Tainan City, \\ Taiwan
}

\begin{abstract}
We reported a rare case of sudden onset of severe but reversible suprachoroidal air that occurred at the moment of air-fluid exchange in 23-gauge vitrectomy. A 31-year-old male patient presented with a large break at 10-11 o'clock and high bullous, nearly total retinal detachment. $\mathrm{He}$ underwent first surgery with silicon oil injection at the end of the surgery. He was arranged to have a second surgery for silicon oil removal through pars plana vitrectomy which was performed smoothly at first. While switching to another mode of air-fluid exchange to clean the residual emulsified oil droplets, surgical view disappeared completely and was suddenly replaced with severe and total suprachoroidal air, which fortunately resolved within 3 days without any other severe complications. Keywords: suprachoroidal air, choroid detachment, vitrectomy, air fluid exchange
\end{abstract}

\section{Introduction}

Small-gauge vitrectomy, a new trend in microsurgery, has become more popular owing to its short surgical time and fast wound healing in vitreoretinal surgeries. Instead of incisional wounds made with a microvitreoretinal knife and sutured at the end of surgery to prevent postoperative wound leak and infection in conventional 20-gauge vitrectomy, there are trocars which are just inserted at an oblique angle to pass through conjunctiva and sclera and into the vitreous directly in 23-gauge vitrectomy without sutures, and these are also easily removed as surgeries are completed. ${ }^{1}$ However, Tarantola et $\mathrm{al}^{2}$ revealed more frequent occurrence of various types of intraoperative choroid detachments (CDs), such as serous, hemorrhagic, gas, and oil, in 23-gauge vitrectomy than in the 20 -gauge procedure.

Uji et $\mathrm{al}^{3}$ reported intraoperative localized suprachoroidal gas after pars plana vitrectomy as gas diffused through the sclerotomy. We would like to report a rare case of sudden onset of severe and total suprachoroidal air that occurred during air-fluid exchange (AFX) after 23-gauge vitrectomy.

\section{Case presentation}

A 31-year-old male patient presented with a large break at 10-11 o'clock and high bullous, nearly total retinal detachment with proliferative vitreoretinopathy grade II and macula off. His best-corrected visual acuity was 20/20 in right eye and 1/20 in left eye with a history of amblyopia. After pars plana vitrectomy, scleral buckle, epiretinal membrane peeling, AFX, endolaser, and silicon oil injection, his left eye vision was at 2/20 for nearly 1 year. However, silicon oil emulsified, leading to reduced vision in left eye later.
Correspondence: Kai-Ling Peng Department of Ophthalmology, Chi Mei Medical Center, No. 901, Zhonghua Road, Yongkang District, Tainan City 710 , Taiwan

Tel +886 6281 28II ext 57247

Email caropk165@gmail.com 
Silicon oil was removed through 23-gauge pars plana vitrectomy which was performed smoothly at first. While clearing the emulsified oil droplets under AFX, multiple gas bubbles fluttered continuously on the posterior surface of the lens preventing a clear view of the fundus using a wide field lens. The view of the fundus was regained after balanced salt solution reinfusion, but it soon disappeared suddenly without red reflex during switching to the second AFX mode (Figure 1). The scleral and conjunctival wounds were closed with patching. Two hours after the surgery, severe left eye pain and pneumoconjunctiva with lots of subconjunctival air covering the peripheral cornea were noted (Figure 2).

Pulse therapy (methylprednisolone $250 \mathrm{mg}$ every 6 hours for 3 days) was given immediately. On postoperative day 1 , the severe penumoconjunctiva resolved completely. The intraocular pressure was $8 \mathrm{mmHg}$ in left eye. The large area of the $\mathrm{CD}$ showed a convex shape in the periphery and had an irregular surface over the posterior pole, when observed by indirect ophthalmoscopy. Brightness scan (B-scan) showed a

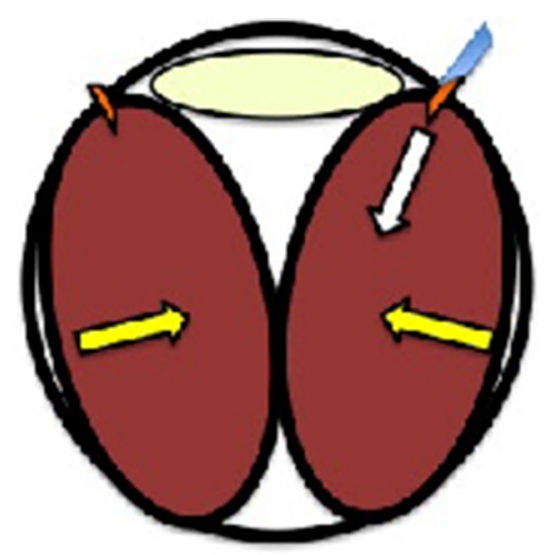

Figure I This illustration shows that the tip of the trocar retracted into the suprachoroidal space.

Notes: The air moved through the infusion cannula and pumped into the suprachoroidal space (white arrow) to lead to a severe and total suprachoroidal air (yellow arrows) without red reflex immediately (brown color: total air CD, light yellow color: lens).

Abbreviation: $\mathrm{CD}$, choroid detachment.

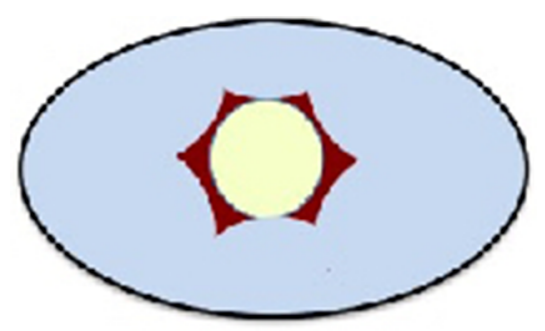

Figure 2 The illustration displays severe pneumoconjunctiva with lots of subconjunctival air (light blue color) covering the peripheral cornea and blocking diffusion of suprachoroidal air through the gap of the sutured sclerotomies (brown color: iris, light yellow color: pupil). higher $\mathrm{CD}$ over the upper area with a shallower one over the lower area on vertical view (Figure 3A). On postoperative day 2, only upper peripheral CD with flat but thickened choroid over the posterior pole appeared on horizontal view from B-scan (Figure 3B). On postoperative day 3, his left vision improved to $2 / 20$ with correction, the same as it was after his previous surgery. $\mathrm{B}$-scan showed that $\mathrm{CD}$ was completely resolved, even the residual smaller one over anterior upper area (Figure 3C). His left vision maintained at $2 / 20$ without other complications during follow-up for 3 months.

\section{Discussion}

CD whose potential space is full of serous fluid, hemorrhage, air or gas between the choroid and sclera may occur during operations or even postoperatively with various causes. The incidence of intraoperative serous CD elucidated recently by Stein et $\mathrm{al}^{4}$ was $0.4 \%-0.5 \%$ in conventional 20 -gauge vitrectomy among Medicare beneficiaries. In contrast, Ooto et $\mathrm{al}^{5}$ reported that the rate of intraoperative choroidal effusions was $1.8 \%$ in 23 -gauge vitrectomy, higher than that of Stein et $\mathrm{al}^{4}$ who used 20 -gauge vitrectomy.

Tarantola et $\mathrm{al}^{2}$ and Ooto et $\mathrm{al}^{5}$ both agreed that the main reason for intraoperative serous choroid detachment was the infusion cannula retraction into the suprachoroidal space ${ }^{2,4}$ where fluid, air, gas, or silicon oil is directly infused. A more oblique and flatter insertion of the trocar with a longer scleral tunnel leads to better postoperative wound sealing and apposition. However, the longer the tunnel inside the sclera is, the shorter the trocar that is exposed into vitreous cavity. Anything that changes the relative position between the sclera and trocar will lead to retraction of the trocar into the suprachoroidal space, further resulting in various types of $\mathrm{CD}$.

Since a vitrectomy machine (Constellation) would keep constant intraocular pressure even when switching to the mode of AFX, we inferred that the air rushed into vitreous cavity after fluid infusion may result in mild retraction of the trocar, so-called the action and reaction. Multiple gas bubbles that fluttered around in the first AFX may be a sign of the retraction of the trocar tip near the residual vitreous and surrounding tissue. After the second switch of the AFX, the trocar tip was again retracted and buried into the suprachoroidal space with severe suprachoroidal air occurring without red reflex.

Suprachoroidal air would resolve spontaneously through diffusion from the posterior pole to the anterior portion when the patient is placed in a supine position and from the lower to the upper portion when the patient is in an upright posture. Distribution of the air through the sclerotomy wound 


\section{A}

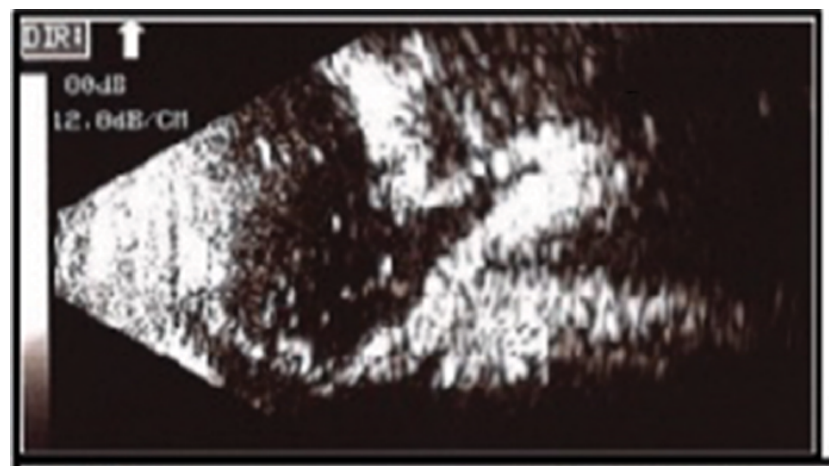

\section{B}
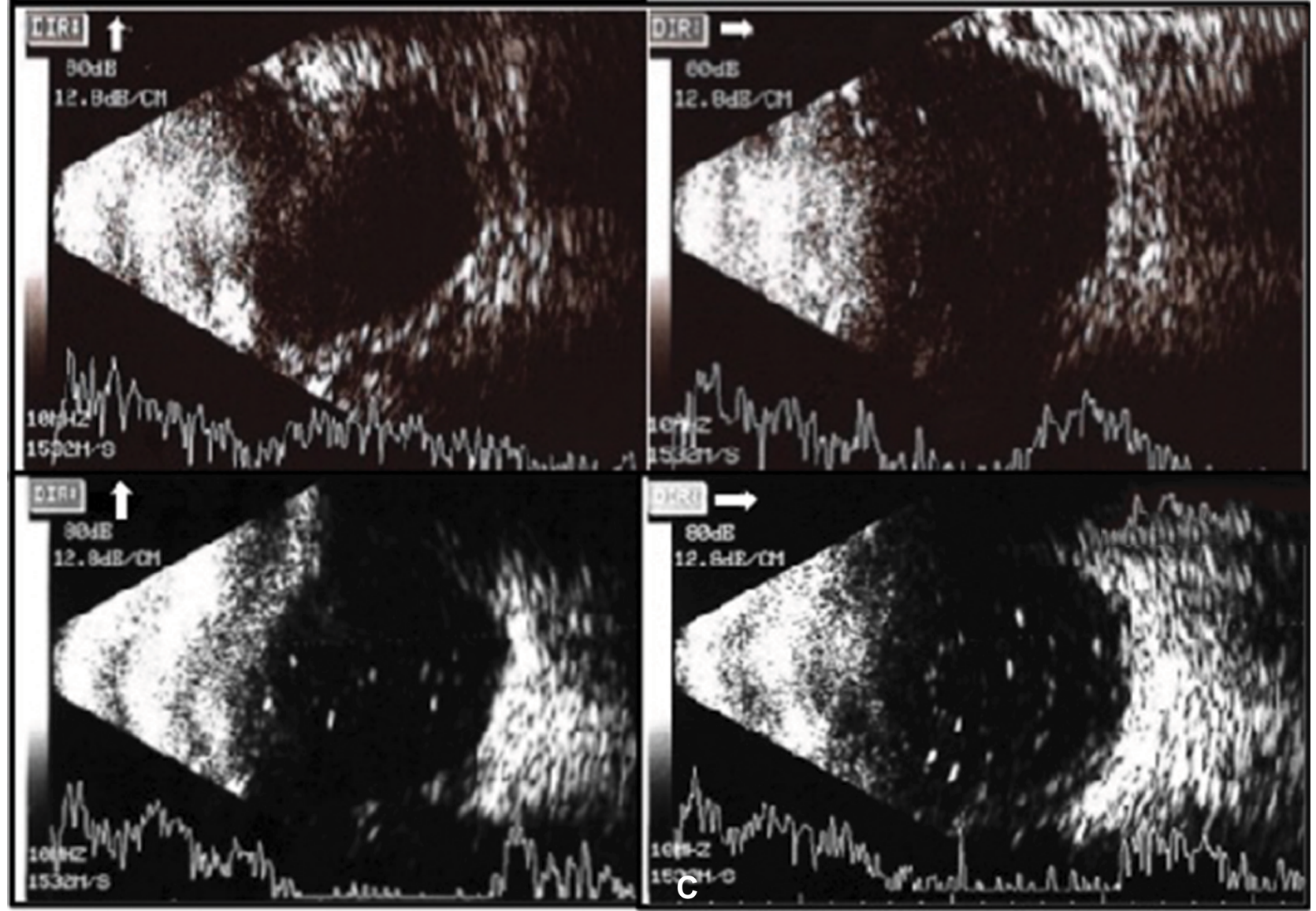

Figure 3 (A) B-scan showed higher CD over the upper area with a shallower one over the lower area on vertical view. (B) On postoperative day 2, just the upper peripheral $C D$ with an obvious flat but thick choroid over the posterior pole appeared on horizontal view from B-scan. (C) B-scan showed the CD totally resolving from the posterior pole to the periphery, with a residual one over the upper area more anteriorly.

Abbreviations: B-scan, brightness scan; $C D$, choroid detachment.

to the subconjuctival space leads to pneumoconjunctiva, which might further diffuse through the slit of the sutured conjunctival wounds. Uji et $\mathrm{al}^{3}$ reported that it took more than 10 days for suprachoroidal gas to totally resolve with flushing of $1.5 \mathrm{~mL} 100 \%$ SF6, a long-lasting gas. Although severe and total suprachoroidal air occurred even without red reflex in our case, it resolved more quickly, in 3-4 days, with vision recovery. Pulse therapy was meant to induce less neuronal cell swelling after this significant complication. Further research is needed to verify if air in the choroidal space would lead to any microstructural damage such as compromising choroidal vasculature and crushing choroidal tissues or retinal neuronal cells.

\section{Conclusion}

Any unusual sign during AFX, such as air fluttering at the posterior surface of lens, must be a sign or an alert. Rechecking the position of infusion trocar when switching the modes may be necessary. Although the severe suprachoroidal air resolved spontaneously without vision deterioration 
in our patient, we should do our best to avoid this severe complication.

\section{Acknowledgment}

Written informed consent was obtained from the patient for publication of this case report and accompanying images.

\section{Author contributions}

Chien-Jen Lin drafted the manuscript. Kai-Ling Peng was responsible for the clinical management of the patient, the design of the case report, and revision of the manuscript. All authors contributed toward data analysis, drafting and critically revising the paper and agree to be accountable for all aspects of the work.

\section{Disclosure}

The authors report no conflicts of interest in this work.

\section{References}

1. Lopez-Guajardo L, Pareja-Esteban J, Teus-Guezala MA. Oblique sclerotomy technique for prevention of incompetent wound closure in transconjunctival 25-gauge vitrectomy. Am J Ophthalmol. 2006;141(6): 1154-1156.

2. Tarantola RM, Folk JC, Shah SS, et al. Intraoperative choroidal detachment during 23-gauge vitrectomy. Retina. 2011;31(5):893-901.

3. Uji A. Suprachoroidal gas injection as a complication of pars plana vitrectomy confirmed by computed tomography. Clin Ophthalmol. 2012;6:533-536.

4. Stein JD, Zacks DN, Grossman D, Grabe H, Johnson MW, Sloan FA. Adverse events after pars plana vitrectomy among medicare beneficiaries. Arch Ophthalmol. 2009;127(12):1656-1663.

5. Ooto S, Kimura D, Itoi K, et al. Suprachoroidal fluid as a complication of 23-gauge vitreous surgery. Br J Ophthalmol. 2008;92(10):1433-1434.
International Medical Case Reports Journal

\section{Publish your work in this journal}

The International Medical Case Reports Journal is an international, peer-reviewed open-access journal publishing original case reports from all medical specialties. Previously unpublished medical posters are also accepted relating to any area of clinical or preclinical science. Submissions should not normally exceed 2,000 words or

\section{Dovepress}

4 published pages including figures, diagrams and references. The manuscript management system is completely online and includes a very quick and fair peer-review system, which is all easy to use. Visit http://www.dovepress.com/testimonials.php to read real quotes from published authors.

Submit your manuscript here: https://www.dovepress.com/international-medical-case-reports-journal-journal 